\title{
Music therapy: an alternative approach in healthcare
}

\section{Opinion}

Music is a magical medium which can potentially delight all our senses and inspire every fibre of our being by bringing us comfort, joy and can be effectively used to alter state of mind, body and soul to promote holistic well being. Music therapy is a no medicine, non invasive, low cost, effective alternative to holistic well being. It helps us to de-stress, rejuvenate and energize in nature's most beautiful way. Use of Indian music for care and cure is age old. The knowhow, though, is not widely accessible to common man today. We aim to bring this wonderful way to wellness to the world, in a scientific manner so that all may believe and benefit from this. Role of music in therapy is not new. Ancient scriptures have well documented evidence of role of music in therapy, which dates back to human civilization of Vedic period. The Indian raagas, mantras and musical notes have been designed in a scientific way to give desired effect to the practitioner as well as the listener, subject to delivery with right frequencies and energy in a prescribed manner. The time at which the raaga is preferably sung, the notes which frequent in the raaga, determines its use in certain conditions. The vibrations, raaga and mantra produce, have a calming effect on the practitioner and the listener.

Historical evidence of music and its employment in education, entertainment, healing and mood management is extensive in writings, frescoes, paintings, leaf and papyrus bindings discovered, documented and preserved across the habitats of all eastern and western ancient civilizations. Twentieth century medical and scientific disciplines commenced efforts post 1930 to codify the value and practice of its therapeutic intervention and acceptance as a noninvasive complementary and alternative medicine. Music, depending on the nature of its delivery and the environment in which it is delivered, connects and communicates with us in different individual ways. In Music Therapy, music is used to address physical, emotional, cognitive, and social needs of individuals. After assessing the strengths and needs of each person, a qualified music therapist provides the indicated treatment including creating, singing, moving to, meditating and/or listening to music. Through musical involvement in the therapeutic context, clients' abilities are strengthened and transferred to other areas of their lives. Music therapy also provides avenues for communication that can be helpful to those who find it difficult to express themselves in words. Research in music therapy supports its effectiveness in many areas such as: overall physical rehabilitation
Volume 5 Issue I - 2018

\section{Govind Saraswati, Sonali Dixit}

Centre for Advanced Research in Indian Music Therapy, India

Correspondence: Sonali Dixit, Centre for Advanced Research in Indian Music Therapy, India, Email sonalidsharma@gmail.com

Received: February 23, 2018 | Published: March 22, 2018

and facilitating movement, increasing people's motivation to become engaged in their treatment, providing emotional support for clients and their families, and providing an outlet for expression of feelings. The understanding of music's role and function in therapy and medicine is undergoing a rapid transformation, based on neuro scientific research showing the reciprocal relationship between studying the neurobiological foundations of music in the brain and how musical behaviour through learning and experience changes brain and behaviour function. Through this research the theory and clinical practice of music therapy is changing more and more from a social science model, based on cultural roles and general well-being concepts, to a neuroscience-guided model based on brain function and music perception. This paradigm shift has the potential to move music therapy from an adjunct modality to a central treatment modality in therapy and rehabilitation.

\section{Acknowledgements}

None.

\section{Conflict of interest}

The author declares there is no conflict of interest. 